\title{
Caractérisation physico-chimique d'un système lotique en région tropicale : la rivière Sô au Sud- Bénin, Afrique de l'Ouest.
}

\author{
${ }^{1}$ KOUDENOUKPO Zinsou Cosme ${ }^{*},{ }^{1} \mathrm{CHIKOU}$ Antoine, ${ }^{1}$ ADANDEDJAN Delphine, ${ }^{1} \mathrm{HAZOUME}$ Rodrigue, \\ ${ }^{2}$ YOUSSAO Issaka, ${ }^{3}$ MENSAH Guy Apollinaire et ${ }^{1}$ LALEYE A. Philippe. \\ 'Laboratoire d'Hydrobiologie et d'Aquaculture de la Faculté des Sciences Agronomiques, Université d'Abomey-Calavi 01 \\ BP 526 Cotonou (Bénin). \\ 2Laboratoire de Biotechnologie Animale et Technologie des viandes, École Polytechnique d'Abomey-Calavi, Université \\ d'Abomey-Calavi 01 BP 526 Cotonou (Bénin). \\ ${ }^{3}$ Institut National des Recherches Agricoles du Bénin (INRAB), Centre de Recherche d'Agonkanmey 01 BP 884 Recette \\ principale Cotonou (Bénin). \\ Auteur correspondant : Email : kkoudenoukpo@yahoo.fr , Tél 0022996431429
}

Original submitted in on $6^{\text {th }}$ January 2017. Published online at www.m.elewa.org on $31^{\text {st }}$ May 2017 https://dx.doi.org/10.4314/jab.v113i1.1

\section{RÉSUMÉ}

Objectif : Faire une caractérisation physico-chimique des eaux de la rivière Sô en vue d'offrir des éléments de base pour une modélisation écologique future du système.

Méthodologie et Résultats : Les données ont été collectées mensuellement pendant 18mois, de Juin 2015 à Novembre 2016 au niveau de douze stations d'échantillonnage. Seize paramètres physico-chimiques dont huit in-situ (température, pH, transparence, profondeur, salinité, oxygène dissous, conductivité électrique et Solides Totaux Dissous) et huit au laboratoire ( $\mathrm{NTK}, \mathrm{NO}_{2}-\mathrm{NO}_{3}-\mathrm{NH}_{4}{ }^{+}, \mathrm{PO}_{4}{ }^{3-}$ ortho, $\mathrm{PO}_{4}^{3}$-total, $\mathrm{Ca}^{2+}$ et $\mathrm{Mg}^{2+}$ ) ont été mesurés. Le test de Kruskal Wallis et de SNK, la matrice de corrélation, la Classification Hiérarchique Ascendante (ACH) et l'Analyse en Composantes Principales (ACP) ont été appliqués à l'ensemble des paramètres mesurés. Les résultats ont permis d'identifier quatre groupes de stations avec $80 \%$ de dissemblance. Le groupe 1 constitué des stations du cours inférieur, présente des eaux fortement minéralisées, les groupes 2 et 3 constitués des stations du cours moyen, témoignent d'une pollution azotée et phosphorée et le groupe 4 constitué des stations du cours supérieur de la rivière présente les caractéristiques des eaux très bien oxygénées et favorable à l'accroissement des espèces aquacoles.

Conclusions et applications : la partie amont de la rivière Sô présente des eaux d'une bonne productivité piscicole tandis que les cours aval et moyen présentent des eaux de fortes teneurs en composés azotés et phosphorés perturbant le biotope ce qui pourrait crée un déséquilibre de la faune aquatique. Ces résultats constituent des données de base pour la mise en place d'un plan de gestion durable de la rivière et son bassin versant.

Mots-clés : Physico-chimie, Pollution, Système lotique, Rivière Sô, Région tropicale. 


\section{ABSTRACT}

Objective: To carry out a physicochemical characterization of the waters of the Sô River in order to provide basic elements for future ecological modeling of the system.

Methodology and Results: Data were collected monthly between June 2015 to November 2016 at twelve sampling stations. Sixteen physico-chemical parameters, eight in situ (temperature, pH, transparency, depth, salinity, dissolved oxygen, electrical conductivity and Dissolved Total Solids) and eight physico-chemical parameters (NTK, $\mathrm{NO}_{2-}, \mathrm{NO}_{3^{-}}, \mathrm{NH}_{4+}, \mathrm{PO}_{3}-\mathrm{PO}_{3}$-total, $\mathrm{Ca} 2+$ and $\mathrm{Mg} 2+$ ) were measured. The Kruskal Wallis and SNK test, correlation matrix, Ascending Hierarchy Classification (HCA) and Principal Component Analysis (PCA) were applied to all measured parameters. The results identified four groups of stations with $80 \%$ dissimilarity. Group 1, consisting of the stations of the lower course, contains highly mineralized waters, groups 2 and 3 consisting of the stations of the middle course, show a nitrogenous and phosphorous pollution and the group 4 consisting of the stations of the upper course of the river presents the characteristics of very well oxygenated waters and favorable to the growth of aquaculture species.

Conclusions and applications : the upstream part of the Sô River has waters for good fish productivity while the downstream and middle courses present waters with high levels of nitrogen and phosphorus compounds disrupting the biotope, which could create an imbalance in fauna aquatic. These results provide basic data for the implementation of a sustainable management plan for the river and its watershed.

Keywords: Physico-chemistry, Pollution, Lotic system, Sô River, Tropical region.

\section{INTRODUCTION}

L'étude de la qualité physico-chimique et l'évaluation du niveau trophique des cours d'eau permet de caractériser les éléments minéraux qui dégradent la qualité des eaux et menacent la vie aquatique (Mama et al., 2011). C'est la concentration de ces éléments qui détermine la qualité d'une eau et permet de savoir si celle-ci convient ou non à un usage particulier. Ces éléments minéraux sont de nature diverse, à l'état dissout ou en suspension (bicarbonates, sulfates, sodium, calcium, magnésium, potassium, azote, phosphore, aluminium, fer.). Ils proviennent du sol et du soussol, de la végétation et de la faune, des précipitations et des eaux de ruissellement drainant le bassin versant, ainsi que des processus biologiques, physiques et chimiques ayant lieu dans le cours d'eau lui-même. À ces substances d'origine naturelle peuvent s'ajouter des produits découlant de la présence humaine (phosphore, azote et microorganismes contenus dans les eaux usées domestiques) ou des activités industrielles et agricoles (substances toxiques, métaux lourds, pesticides). Ce qui n'est pas sans incidences sur les écosystèmes naturels (Dèdjiho, 2013). Au Bénin, la rivière Sô, est un cours d'eau fortement anthropisé avec l'augmentation de la population lacustre. Cette anthropisation de la rivière se justifie d'une part, par les intenses activités agricoles nécessitant des engrais chimiques qui se développent tout au long de son bassin versant et d'autre part, par d'importantes quantités de branchages servant à la construction de piège à poissons qui y sont quotidiennement déversées par les pêcheurs dans le cadre des activités halieutiques. De plus, la rivière et sa berge sont devenues un réceptacle de déchets solides et liquides jetés par les populations, de nombreuses déjections de porcs et des bœufs et enfin du trafic frauduleux des hydrocarbures (pétrole essence, gasoil, huile à moteur) (Kiossa, 2011). Dans ces conditions d'absence totale de mesure d'hygiène et d'assainissement, les risques écotoxicologiques sont très élevées, la qualité des eaux est compromise et la faune aquatique est fortement menacée. II urge donc que cette étude soit menée afin d'offrir les premières données sur la qualité des eaux de cette rivière, car la seule étude officielle menée sur cette rivière concerne celle de Adité et al., (2006) portant sur la biologie et l'écologie d'une espèce de poisson Heterotis niloticus.La présente étude vise donc à caractériser les eaux de cette rivière pour avoir les éléments de base pour sa modélisation écologique et son fonctionnement. 


\section{MATERIEL et METHODES}

Milieu d'étude : La rivière Sô est comprise entre $6^{\circ} 24^{\prime}$ et $6^{\circ} 32^{\prime}$ Latitude Nord et $2^{\circ} 27^{\prime}$ et $2^{\circ} 30^{\prime}$ Longitude Est. Elle est située dans la commune de Sô-Ava, commune à laquelle elle doit son nom. D'une longueur de $84,4 \mathrm{Km}$, la rivière Sô prend sa source dans le lac Hlan et est reliée au fleuve Ouémé par des marigots. Cette rivière est l'une des anciens bras du fleuve Ouémé, qui entre temps s'en est détachée, et qui déverse ses eaux au Nord-Ouest du lac Nokoué au niveau de la cité lacustre de Ganvié (Lalèyè, 1995). Tout au long du bassin de cette rivière, les riverains pratiquent d'importantes activités agricoles (patates, manioc, maïs et cultures) nécessitant l'usage des engrais et l'élevage des porcs et bœufs laissées en divagation sur les berges. De même pour leurs activités de pêche, de nombreux branchages sont utilisées pour la réalisation des acadjas qui abondent la rivière et enfin le trafic frauduleux des hydrocarbures qui s'observe quotidiennement sont autant d'activités anthropiques qui se développent dans ce milieu.

Choix des stations : Afin de rechercher la variabilité des paramètres du milieu, la rivière a été subdivisée suivant la stratification longitudinale proposée par Tachet et al., (2000) et Ahouansou (2011). Ainsi, d'amont en aval de la rivière, douze stations d'échantillonnage ont été retenues. Les stations ont été choisies en fonction de l'accessibilité à la station, la présence ou l'absence d'agglomérations urbaines, l'existence d'activités agricoles ou d'un gradient de pollution, la diversité du biotope et la présence ou l'absence de la végétation bordière. Ces caractéristiques permettent d'affiner le portrait spatial de la qualité de l'eau le long de la rivière. Le tableau 1 présente les coordonnées géographiques des stations retenues par secteur. La localisation géographique du bassin de la rivière et des stations d'études est donnée par la figure 1.

Tableau 1 : Sites de prélèvement des eaux de la rivière Sô

\begin{tabular}{|c|c|c|}
\hline Sites & Noms & Coordonnées géographiques \\
\hline ST1 & Vêky & N0716'98.4", E004³5’82.2"' \\
\hline ST2 & Sindomey & N07¹5'84.3", E004³2'50.0" \\
\hline ST3 & Dogodo & N07 18'40.2", E004³3’56.3"' \\
\hline ST4 & Ahomey-Gblon & N07²2'65.2" ; E004³4'02.2" \\
\hline ST5 & Ahomey-Ounmey & N07 ${ }^{\circ} 25^{\prime} 40.3^{\prime \prime} ;$ E004³3'79.1" \\
\hline ST6 & Ahomey-Lokpo & N07 $27^{\circ} 28.3^{\prime \prime} ;$ E004³3'17.7'” \\
\hline ST7 & Zoungomey & N07²9'86.2" ; E004³3'78.3" \\
\hline ST8 & Kinto Oudjra & N07 $33^{\prime} 84.3^{\prime \prime} ;$ E004 $35^{\prime} 81.2^{\prime \prime}$ \\
\hline ST9 & Togbota & N07 $39^{\prime} 40.6^{\prime \prime} ;$ E004³4'81.3" \\
\hline ST10 & Tota & N0740'98.2" ; E004³8'99.8'” \\
\hline ST11 & Rhlampa & N074'45.4" ; E004³7’47.9"' \\
\hline ST12 & Djigbé-Ovo & N0752'96.2" ; E004³5’99.8" \\
\hline
\end{tabular}




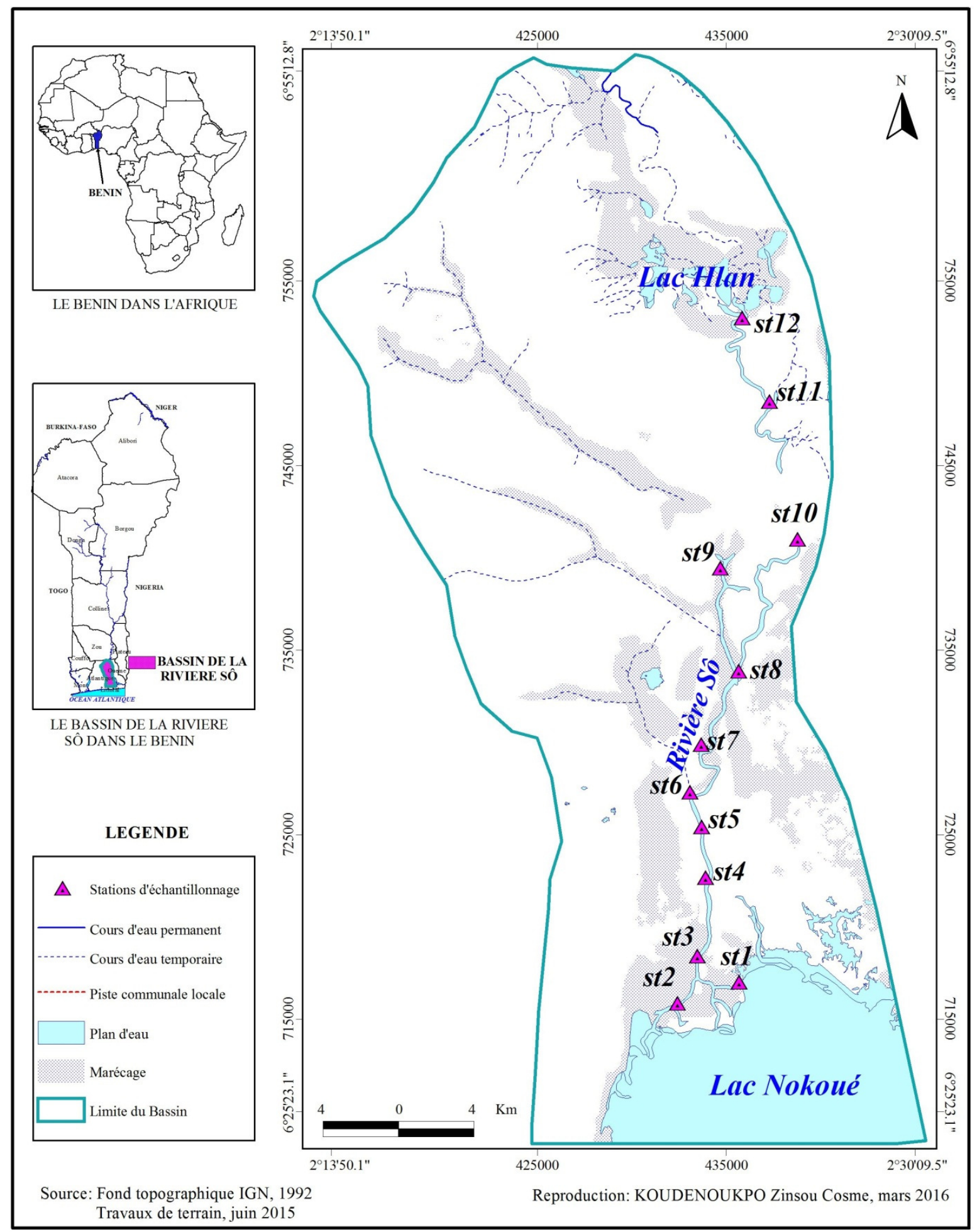

Figure 1: Carte du bassin de la rivière Sô et des stations d'échantillonnage.

St1 = Vêky, St2= Sindomey, St3= Dogodo, St4= Ahomey-Gblon, St5= Ahomey-Ounmey, St6= Ahomey-Lokpo, St7=Zoungomey, St8= Kinto-Oudjra, St9= Togbota, St10= Tota, St11 = Rhlampa et St12= Djigbé-Ovo 

région tropicale : la rivière Sô au Sud-Bénin, Afrique de l'Ouest.

Échantillonnage: Les données ont été prises mensuellement pendant 18 mois (Juin 2015 à Novembre 2016). Les mesures et prélèvements des eaux ont eu lieu entre $06 \mathrm{~h} 30$ et $10 \mathrm{~h} 45$. Lors de chaque campagne et à chacune des stations décrites plus haut, la transparence et la profondeur ont été mesurées à l'aide d'un disque de Secchi; un multi paramètre, Model SX736 $\mathrm{pH} / \mathrm{mV} /$ Conductivity/DO Meter, possédant deux sondes a permis de mesurer le $\mathrm{pH}$, la température l'oxygène dissous, la salinité, le TDS et la conductivité électrique. Après ces mesures, les prélèvements d'eau ont été faits dans des bouteilles en polyéthylène à double bouchage de contenace $1000 \mathrm{~mL}$, conservés dans une glacière à $4^{\circ} \mathrm{C}$ puis ramener au laboratoire. Au laboratoire, les paramètres chimiques telque le calcium, magnésium, azote total, nitrite, nitrate, ammonium, orthophosphate et le phosphore total ont été dosés par chromatographie ionique à l'aide d'un Chromatographe ionique DIONEX ICS-1000 respectivement au Nesler, Diazotation, Réduction au Cd, Nessler et Acide ascorbique selon Rodier (2009). En effet, le dosage par chromatographie ionique est une technique analytique qui permet l'analyse qualitative (par séparation des espèces présentes) et quantitative des espèces ioniques présentes dans un échantillon liquide dépourvu de Matières en suspension (Rodier, 2009).

Analyses statistiques : La base de données qui a servi aux analyses et traitements statistiques est constituée de 16 paramètres physico-chimique*12stations ${ }^{*} 18$ mois. La matrice de corrélation a été établie pour montrer les coefficients de corrélation entre les différents paramètres

\section{RESULTATS et DISCUSSION}

Environnement physico-chimique des eaux: Les tableaux 2 et 3 présentent respectivement les variations spatiales et saisonnières des valeurs moyennes des paramètres physico-chimiques des eaux de la rivière Sô. Le test de Kruskal-Wallis réalisé montre que les paramètres varient plus sur le plan saisonnier que sur le plan spatial. Parmi les seize paramètres étudiés, seuls la profondeur, la conductivité, le TDS, la salinité, l'oxygène dissous et les composés phosphorés varient très significativement d'un secteur de la rivière à un autre. Par contre sur le plan saisonnier, tous les paramètres montrent des variations hautement significatives $(p<$ $0,0001)$ à l'exception de la température $(p<0,05)$.

Matrice de corrélation: Le tableau 4 montre les coefficients de corrélations entre les différents paramètres mesurés. Le coefficient de corrélation détermine la relation entre deux variables et mesure l'intensité de ce lien. II varie entre -1 et +1 . Lorsqu'il est égal à 1 , il indique un lien parfait entre les variables et lorsqu'il est égal à 0 , il indique une absence de lien. Le signe $(+)$ signifie que la relation est proportionnelle alors que le signe (-) signifie que la relation est inversement proportionnelle. Le calcul du coefficient de corrélation permet d'avoir une idée sur les éventuelles relations entre paramètres: origine commune, distribution homogène, comportement identique vis-à-vis des processus physico-chimiques, fractionnement similaire, influence de l'un sur l'autre. Le test de Shapiro-Wilk a été appliqué pour vérifier si les paramètres physico-chimiques de la rivière suivent une distribution normale. Le test de Kruskal-Wallis a été utilisé pour tester la variabilité des paramètres entre les mois et les stations définies. Quant au test de SNK, il a permis de faire les comparaisons deux à deux (d'un secteur à un autre, et d'une saison à une autre). Ensuite la typologie abiotique a été réalisée, grâce à une Classification Hiérarchique Ascendante (ACH) telle que préconisée par Belkhiri et al., (2010) sur les valeurs moyennes des différents paramètres afin de ressortir les différents groupes de stations. Afin d'obtenir une meilleure affectation des paramètres à leur groupe, une Analyse en Composantes Principales a été enfin réalisée sur les valeurs moyennes des différents groupes obtenus après la classification. Toutes les analyses statistiques effectuées ont été réalisées avec le logiciel $R$ version 2.15.3 muni du package FactoMineR.

mesurés. On remarque une corrélation négative et significative entre la profondeur des eaux et les teneurs en nitrites, nitrates et l'azote total. Quand la profondeur des eaux augmente, les teneurs de ces composés azotés diminuent. Par ailleurs, la température et le $\mathrm{pH}$ des eaux sont positivement corrélés à la conductivité, le TDS, la salinité, le phosphore total, le calcium et le magnésium d'une part et négativement corrélés à l'oxygène d'autre part. On note également une corrélation négative entre l'oxygène et les teneurs en nitrites, nitrates, ammonium, phosphore total, calcium et le magnésium. Enfin, une forte corrélation positive est observée entre l'ammonium et l'orthophosphate. Cette corrélation est hautement significative. Il en est de même entre le phosphore total, le calcium et le magnésium. 
Koudenoukpo et al., J. Appl. Biosci. 2017 Caractérisation physico-chimique d'un système lotique en région tropicale : la rivière Sô au Sud-Bénin, Afrique de l'Ouest.

Tableau 2 : Variations spatiales des paramètres physico-chimiques de la rivière Sô

\begin{tabular}{|c|c|c|c|c|}
\hline Saisons & $\mathrm{Cl}$ & CM & CS & Significativité \\
\hline Trans & $0,75^{\mathrm{a}} \pm 0,44$ & $0,85^{\mathrm{a}} \pm 0,44$ & $2,31^{b} \pm 9,25$ & * \\
\hline Prof & $3,74^{a} \pm 1,66$ & $3,59^{b} \pm 0,68$ & $3,35^{a} \pm 1,48$ & * \\
\hline Temp & $28,60^{a} \pm 1,64$ & $27,98^{a} \pm 1,71$ & $27,90^{\mathrm{a}} \pm 1,37$ & NS \\
\hline pH & $7,32^{a} \pm 0,55$ & $7,10^{a} \pm 0,61$ & $7,07 \mathrm{a} \pm 0,53$ & NS \\
\hline Cond & $2929,10^{a} \pm 4403,19$ & $1530,80^{b} \pm 2257,12$ & $611,93^{c} \pm 653,89$ & * \\
\hline TDS & $1487,11^{a} \pm 2618,68$ & $217,70^{b} \pm 273,27$ & $129,49^{c} \pm 318,30$ & * \\
\hline Sal & $1,61^{\mathrm{a}} \pm 1,42$ & $0,35^{b} \pm 0,44$ & $0,40^{c} \pm 0,63$ & $* * *$ \\
\hline 02 & $6,40^{a} \pm 2,67$ & $7,42^{b} \pm 2,71$ & $7,54^{b} \pm 2,53$ & * \\
\hline $\mathrm{NO}_{2}^{-}$ & $0,14^{a} \pm 0,13$ & $0,15^{\mathrm{a}} \pm 0,19$ & $0,13^{a} \pm 0,18$ & NS \\
\hline $\mathrm{NO}_{3}^{-}$ & $2,22^{a} \pm 3,08$ & $1,93^{a} \pm 2,48$ & $2,19^{a} \pm 3,59$ & NS \\
\hline $\mathrm{NH}_{4}^{+}$ & $1,37^{a} \pm 1,58$ & $1,00^{a} \pm 1,10$ & $0,74^{a} \pm 0,72$ & NS \\
\hline AzoT & $4,39 a \pm 2,48$ & $4,34^{\mathrm{a}} \pm 2,90$ & $4,95^{\mathrm{a}} \pm 3,52$ & NS \\
\hline OrthoP & $0,63^{a} \pm 1,50$ & $0,24^{b} \pm 0,22$ & $0,19 b \pm 0,08$ & * \\
\hline PhosT & $1,64 \mathrm{a} \pm 1,86$ & $1,16^{b} \pm 1,69$ & $1,20^{b} \pm 1,09$ & * \\
\hline $\mathrm{Ca}_{2}{ }^{+}$ & $50,08^{a} \pm 66,59$ & $21,03^{b} \pm 16,33$ & $18,57^{b} \pm 17,63$ & ** \\
\hline $\mathrm{Mg}_{2}{ }^{+}$ & $69,64^{a} \pm 121,83$ & $20,51^{b} \pm 31,14$ & $18,43^{b} \pm 28,82$ & * \\
\hline
\end{tabular}

$\mathrm{Cl}=$ Cours inférieur $; \mathrm{CM}=$ Cours moyen et $\mathrm{CS}=$ Cours supérieur de la rivière ; Trans= Transparence $;$ Prof $=$ Profondeur ; $\mathrm{pH}=$ Potentiel d'Hydrogène ; O2= Oxygène dissous ; Temp= Température ; Sal= Salinité ; Cond= Conductivité ; TDS= Solides totaux dissous ; $\mathrm{Ca}^{2+}=$ Calcium ; $\mathrm{Mg}^{2+}=$ Magnésium; $\mathrm{NO}_{2}{ }^{-}=$Nitrite; $\mathrm{NO}_{3}{ }^{-}=$Nitrate; $\mathrm{NH}_{4}{ }^{+}=$Ammonium; AzoT= Azote Total; $\mathrm{PO}_{4}{ }^{3-}=$ Orthophosphate; PhosT= Phosphore Total; NS= Non significatif $(p>0,05) ;{ }^{*}=$ Significativité au seuil de $5 \%(p<0,05) ; \quad{ }^{* *}=$ Significativité au seuil de $1 \%(p<0,01)$ et ${ }^{* * *}=$ Significativité au seuil de $1 \% 0(p<0,001)$.

Tableau 3: Variations saisonnières des paramètres physico-chimiques de la rivière Sô

\begin{tabular}{|c|c|c|c|c|c|}
\hline Paramètres & GSP & GSS & PSP & PSS & Significativité \\
\hline Trans & $0,83^{a} \pm 0,40$ & $1,28^{b} \pm 0,41$ & $0,54^{c} \pm 0,14$ & $3,29 d \pm 13,20$ & $* \star *$ \\
\hline Prof & $4,67^{a} \pm 1,45$ & $3,18^{b} \pm 1,19$ & $3,56^{b} \pm 1,42$ & $3,84^{c} \pm 1,16$ & $* * *$ \\
\hline Temp & $28,17^{a} \pm 0,71$ & $28,31^{a} \pm 2,63$ & $28,41^{a} \pm 1,74$ & $27,67^{\mathrm{b}} \pm 0,85$ & * \\
\hline $\mathrm{pH}$ & $7,46^{a} \pm 0,44$ & $6,95^{b} \pm 0,61$ & $6,61^{c} \pm 0,44$ & $7,29 a \pm 0,36$ & $* * *$ \\
\hline Cond & $3139,83^{a} \pm 4121,89$ & $993,83^{b} \pm 822,56$ & $143,98 c \pm 47,62$ & $659,35^{d} \pm 1458,28$ & $* * *$ \\
\hline TDS & $1214,38^{a} \pm 2304,95$ & $41,72^{b} \pm 13,14$ & $65,45^{c} \pm 19,32$ & $504,62^{d} \pm 1171,70$ & $* * *$ \\
\hline Sal & $0,67^{a} \pm 1,02$ & $1,17^{b} \pm 1,20$ & $0,94^{\mathrm{a}} \pm 1,22$ & $0,34^{c} \pm 0,78$ & $* * *$ \\
\hline 02 & $6,14^{\mathrm{a}} \pm 1,40$ & $9,73^{b} \pm 2,91$ & $6,77^{\mathrm{a}} \pm 2,85$ & $5,99 c \pm 1,91$ & $* * *$ \\
\hline $\mathrm{NO}_{2}^{-}$ & $0,09 \mathrm{a} \pm 0,10$ & $0,07^{b} \pm 0,04$ & $0,41^{c} \pm 0,24$ & $0,12^{c} \pm 0,08$ & $* * *$ \\
\hline $\mathrm{NO}_{3}{ }^{-}$ & $0,55^{\mathrm{a}} \pm 0,50$ & $0,58^{a} \pm 0,49$ & $4,35^{b} \pm 4,39$ & $6,05^{c} \pm 2,59$ & $* * *$ \\
\hline $\mathrm{NH}_{4}^{+}$ & $1,09 \mathrm{a} \pm 1,27$ & $1,27^{b} \pm 1,65$ & $0,86^{c} \pm 0,74$ & $0,74^{a} \pm 0,21$ & $* * *$ \\
\hline AzoT & $3,65^{\mathrm{a}} \pm 1,27$ & $3,15^{b} \pm 3,63$ & $6,83^{c} \pm 3,12$ & $6,67^{d} \pm 2,54$ & $* * *$ \\
\hline OrthoP & $0,58^{a} \pm 1,35$ & $0,19 b \pm 0,06$ & $0,22^{b} \pm 0,08$ & $0,16^{c} \pm 0,17$ & $* * *$ \\
\hline Phost & $1,09 \mathrm{a} \pm 0,85$ & $2,41^{b} \pm 2,85$ & $0,56^{c} \pm 1,11$ & $1,09 \mathrm{a} \pm 0,94$ & $* * *$ \\
\hline $\mathrm{Ca}_{2}{ }^{+}$ & $33,46^{a} \pm 27,47$ & $48,19^{b} \pm 72,49$ & $8,02^{c} \pm 3,13$ & $15,42^{d} \pm 17,55$ & $* * *$ \\
\hline $\mathrm{Mg}_{2}^{+}$ & $36,67^{a} \pm 80,30$ & $71,46^{b} \pm 104,85$ & $5,16^{c} \pm 2,00$ & $13,16^{d} \pm 27,17$ & $* * *$ \\
\hline
\end{tabular}

GSP = Grande saison des pluies; GSS = Grande saison sèche $;$ PSP $=$ Petite saison des pluies et PSS $=$ Petite saison sèche. 
Tableau 4 : Matrice de corrélation entre les paramètres physico-chimiques mesurés au niveau de douze stations dans la rivière Sô pendant la période d'étude.

\begin{tabular}{|c|c|c|c|c|c|c|c|c|c|c|c|c|c|c|c|c|}
\hline & Trans & Prof & Temp & $\mathrm{pH}$ & Cond & TDS & Sal & 02 & $\mathrm{NO}_{2}-$ & $\mathrm{NO}_{3} \cdot$ & $\mathrm{NH}_{4}{ }^{+}$ & AzoT & $\mathrm{PO}_{4}^{3-}$ & PhosT & $\mathrm{Ca}^{2+}$ & $\mathrm{Mg}^{2+}$ \\
\hline Trans & 1 & $-0,18$ & $-0,48$ & $-0,06$ & $-0,29$ & $-0,28$ & $-0,08$ & 0,12 & 0 & 0,31 & $-0,21$ & $0,61^{*}$ & $-0,14$ & $-0,13$ & $-0,18$ & $-0,22$ \\
\hline Prof & & 1 & 0,38 & $-0,13$ & $-0,07$ & $-0,17$ & $-0,17$ & 0,07 & $-0,69^{*}$ & $-0,6^{*}$ & $-0,11$ & $-0,51$ & $-0,13$ & $-0,33$ & $-0,17$ & 0,12 \\
\hline Temp & & & 1 & 0,56 & $0,63^{*}$ & $0,68^{*}$ & $0,58^{*}$ & $-0,52$ & $-0,18$ & 0,02 & 0,05 & $-0,51$ & $-0,09$ & 0,53 & $0,62^{*}$ & $0,69^{*}$ \\
\hline $\mathrm{pH}$ & & & & 1 & $0,76^{* *}$ & $0,73^{* *}$ & $0,86^{* * *}$ & $-0,73^{* *}$ & 0,40 & $0,59^{*}$ & 0 & $-0,06$ & $-0,18$ & $0,86^{* \star *}$ & $0,84^{* * *}$ & 0,71 \\
\hline Cond & & & & & 1 & $0,92^{* * *}$ & 0,81 & $-0,78$ & 0,25 & 0,24 & 0,19 & $-0,01$ & 0,01 & $0,68^{*}$ & $0,72^{* *}$ & $0,80^{* *}$ \\
\hline TDS & & & & & & 1 & 0,89 & $-0,6$ & 0,30 & 0,53 & 0,42 & $-0,21$ & 0,30 & $0,91^{* * *}$ & $0,94^{\star * *}$ & $0,86^{* * *}$ \\
\hline Sal & & & & & & & 1 & $-0,73$ & 0,26 & $0,63^{*}$ & 0,16 & 0,05 & 0.04 & $0,92^{* * *}$ & $0,94^{* * *}$ & $0,91^{* * *}$ \\
\hline 02 & & & & & & & & 1 & $-0,26$ & $-0,21$ & $-0,24$ & 0,12 & $-0,13$ & $-0,56$ & $-0,58^{*}$ & $-0,61^{*}$ \\
\hline $\mathrm{NO}_{2}-$ & & & & & & & & & 1 & $0,58^{\star}$ & 0,21 & 0,03 & 0,09 & 0,46 & 0,37 & 0 \\
\hline $\mathrm{NO}_{3}-$ & & & & & & & & & & 1 & $-0,18$ & 0,40 & $-0,2$ & $0,78^{\star *}$ & $0,70^{*}$ & 0,39 \\
\hline $\mathrm{NH}_{4}^{+}$ & & & & & & & & & & & 1 & $-0,37$ & $0,96^{\star * *}$ & 0,14 & 0,15 & 0,19 \\
\hline AzoT & & & & & & & & & & & & 1 & $-0,25$ & 0,02 & $-0,08$ & $-0,05$ \\
\hline $\mathrm{PO}_{4}^{3-}$ & & & & & & & & & & & & & 1 & 0,025 & 0,02 & 0,09 \\
\hline PhosT & & & & & & & & & & & & & & 1 & $0,96^{\star * *}$ & $0,79^{* *}$ \\
\hline $\mathrm{Ca}^{2+}$ & & & & & & & & & & & & & & & 1 & $0,88^{* * *}$ \\
\hline $\mathrm{Mg}^{2+}$ & & & & & & & & & & & & & & & & 1 \\
\hline
\end{tabular}


Koudenoukpo et al., J. Appl. Biosci. 2017 Caractérisation physico-chimique d'un système lotique en région tropicale : la rivière Sô au Sud-Bénin, Afrique de l'Ouest.

Typologie abiotique des stations : La figure 2 présente les résultats de la Classification Hiérarchique Ascendante effectuée sur les caractéristiques physico-chimiques des stations étudiés.

\section{Cluster Dendrogram}

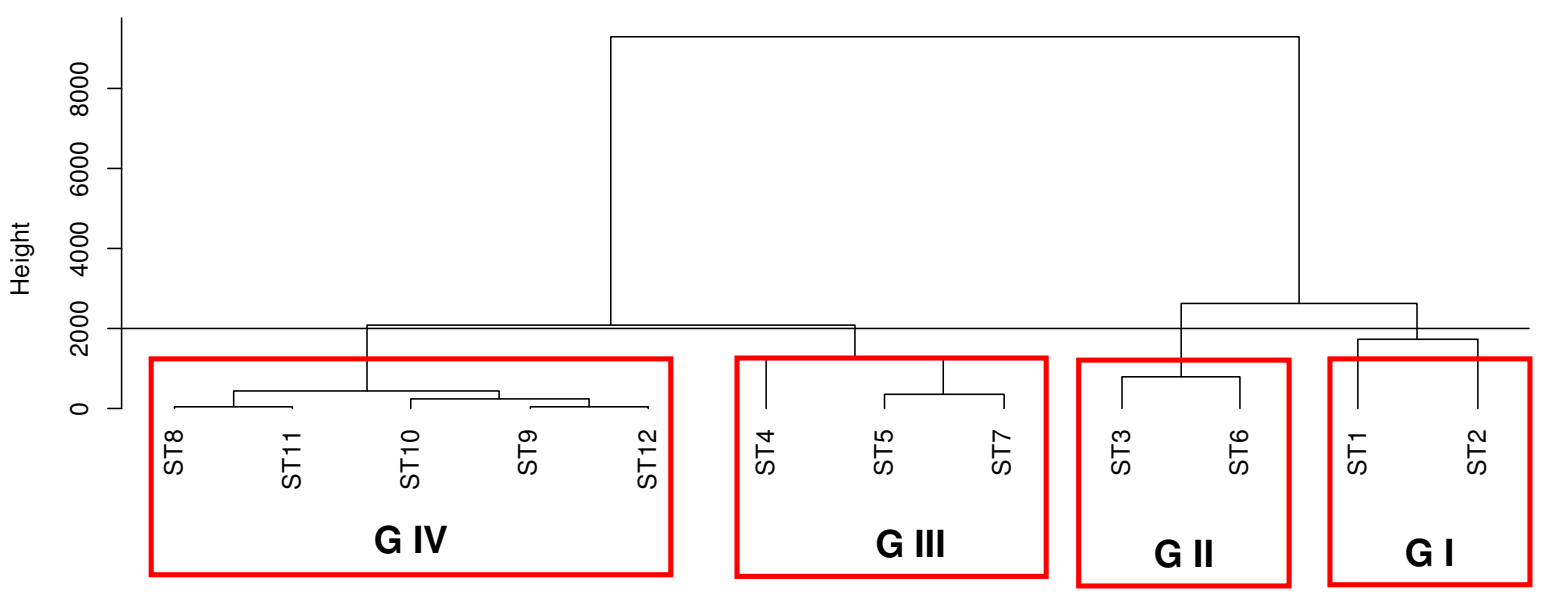

Figure 2 : Dendrogramme représentant la Classification Hiérarchique Ascendante (ACH) des 12 stations d'étude avec un $R^{2}=80 \%\left(R^{2}=10000-2000\right)$.

De l'analyse de la figure 2, quatre (4) groupes de stations se distinguent avec plus $80 \%$ de dissemblance :

- Groupe I : constitué essentiellement des stations 1 et 2 .

- $\quad$ Groupe II : composé des stations 3 et 6

- $\quad$ Groupe III : rassemble les stations 4, 5 et 7

- $\quad$ Groupe IV : composé des stations 8, 9, 10, 11 et 12.

Caractéristiques des groupes: Cette typologie abiotique montre que les groupes I et II sont constitués des stations du cours inférieur, le groupe III constitué des stations du cours moyen et enfin le groupe IV est constitué des stations du cours supérieur. L'analyse en composante principale réalisée à partir des valeurs moyennes des caractéristiques physico-chimiques de ces groupes obtenues après la classification hiérarchique ascendante, révèle que les deux premiers axes concentrent $87,24 \%$ des informations de départ (Figure 3 ), ce qui est suffisant pour garantir une précision d'interprétation car l'axe 3 concentre seulement à elle seul $12,76 \%$ des informations.

\section{Variables factor map (PCA)}

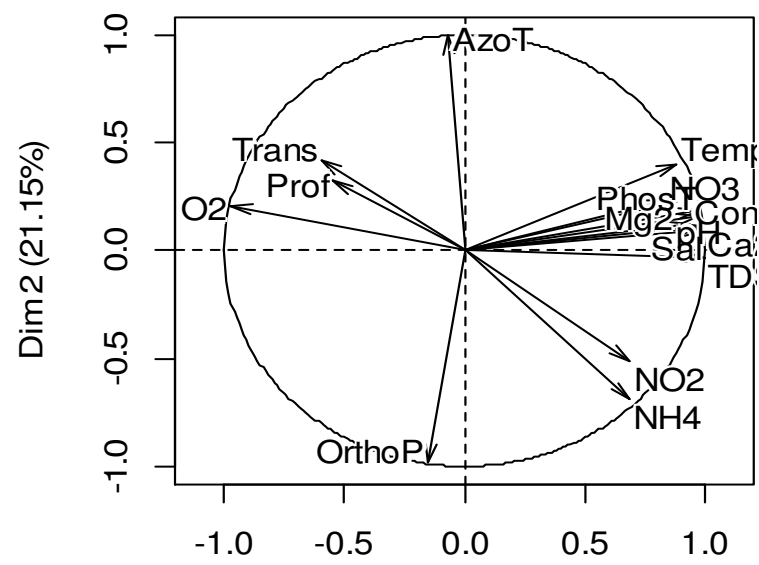

$\operatorname{Dim} 1(66.09 \%)$

Figure 3 : Analyse en Composantes Principales réalisée à partir des paramètres physico-chimiques des stations. 

région tropicale : la rivière Sô au Sud-Bénin, Afrique de l'Ouest.

Le cercle de corrélation montre que l'axe 1 est corrélé positivement à la température, conductivité électrique, $\mathrm{pH}$, calcium, magnésium, $\mathrm{pH}$, salinité, TDS, nitrites, nitrates et au phosphore total par contre il est corrélé négativement à la transparence, profondeur et à l'oxygène dissous. L'axe 2 quant à lui, est expliqué positivement à l'azote total et négativement à l'orthophosphate et l'ammonium. La projection des paramètres physico-chimiques et des groupes de stations dans le système d'axe 1 et 2 défini par l'ACP indique que :

\section{DISCUSSION}

Les produits chimiques libérés dans l'environnement sous l'effet de processus naturels ou par suite d'activités anthropiques peuvent pénétrer dans les écosystèmes aquatiques et s'intégrer dans les matières en suspension, ce qui représente un énorme danger pour les organismes aquatiques (Noumon et al., 2015). Les températures enregistrées sur la rivière Sô sont comprises dans la fourchette 27,41 à $28,94^{\circ} \mathrm{C}$ et sont semblables à celles trouvées par Zinsou et al., (2016) dans le Delta de l'Ouéme. Elles sont relativement proches des valeurs obtenues par Tchakonté et al., (2015) dans une étude réalisée sur cinq rivières au Cameroun, mais ne présentent pas d'importantes variations entre elles et ne peuvent, par conséquent, pas présager de l'existence d'un risque de croissance accélérée des microorganismes et des algues pouvant conduire à une dégradation prononcée du milieu récepteur. Selon Pouomogne (1998), ces valeurs sont favorables à une bonne croissance des espèces piscicoles couramment élevées. Le $\mathrm{pH}$, quant à lui présente des valeurs se situant dans la gamme de 6,92 et 7,57 avec une moyenne de 7,16 . Ces mêmes résultats ont été trouvés par Onana (2014) dans les cours d'eau Mboppi et Simbi au Cameroun. Ces valeurs proches de la neutralité sont caractéristiques de la majorité des eaux de surface et par conséquent n'indiquent ni pollution acide ni pollution basique des eaux de la rivière. Elles traduisent selon la grille de Beaux (1998) des eaux de bonne qualité et surtout pour l'aquaculture. Par ailleurs, la profondeur de la rivière varie en fonction du point d'échantillonnage à partir duquel elle est mesurée. Moyennement, elle est de 3,55 $\mathrm{m}$ sur l'ensemble de la rivière Sô et sa variation est hautement significative d'une station à une autre. Ces mêmes valeurs ont été retrouvées par Ahouanssou (2011) sur la rivière Pendjari. La profondeur atteint des valeurs jusqu'à $6,17 \mathrm{~m}$ au niveau de la station 3 . Cette valeur élevée se justifie par l'extraction du sable au
-Le cours inférieur représenté par le groupe I est caractérisé par des valeurs élevées de conductivité électrique, température, salinité, pH, TDS, calcium, magnésium, nitrite, nitrate, d'azote total et de phosphore total ;

-Le cours moyen représenté par les groupes 2 et 3 , est caractérisé par des concentrations élevées en orthophosphate, en ammonium, en nitrite et en azote total ;

-Le cours supérieur de la rivière représenté par le groupe 4 est caractérisé par des valeurs élevées d'oxygène, de transparence et de profondeur.

niveau de cette station. La conductivité désigne la propriété qu'a une solution à conduire le courant électrique. Cette mesure permet d'évaluer rapidement le degré de minéralisation d'une eau, c'est à dire la quantité de substances dissoutes ionisées qui y sont présentes. La conductivité électrique moyenne du groupe 1 est de $3885,89 \mu \mathrm{S} / \mathrm{cm}$, ce qui montre que les stations constituant ce groupe sont fortement minéralisées suivant la grille de Nisbet et verneaux (1970). Notons que ces valeurs sont largement supérieures à celles observées par Bonou et Adisso (2002) et à la valeur limite de $500 \mu \mathrm{S} / \mathrm{cm}$ rapportée par Belaud (1987). Les stations du cours supérieur de la rivière, présentent des valeurs élevées de transparence et d'oxygène dissous respectivement de 2,04 $\mathrm{m}$ et 7,66 $\mathrm{mg} / \mathrm{L}$. En effet, la clarté de l'eau a une incidence sur la quantité d'oxygène produit par le milieu. Une eau claire permettra à la lumière de pénétrer davantage et favorisera ainsi la photosynthèse. Ces valeurs montrent que les stations du cours supérieur de la rivière sont très bien oxygénées, l'on peut donc avancer que le cours supérieur de la rivière est en bon état. Ces résultats sont semblables à ceux de Chouti et al., (2010). Ces auteurs indiquent que la teneur en oxygène donne des indications sur la santé en des cours d'eau et permet, entre autres d'évaluer la qualité des habitats des poissons. Les valeurs d'oxygène comprise dans cette gamme est acceptable pour le stade initial et les autres stades de vie dans les écosystèmes tropicaux conformément aux recommandations canadiennes pour la qualité des eaux (CCME, 2011). Les valeurs d'oxygène trouvées sont accompagnées par une augmentation des solides totaux dissous traduisant des conditions plus favorables à la dégradation de la charge organique. Ce paramètre fait partie intégrante du métabolisme de l'écosystème. Les poissons et autres animaux en consomment pour le maintien de leur métabolisme (phénomène de respiration cellulaire). En outre, l'ammoniaque représenté souvent 

région tropicale : la rivière Sô au Sud-Bénin, Afrique de l'Ouest.

sous forme d'ion ammonium ( $\mathrm{NH}^{+}$) présente des valeurs faibles comprise entre 0,71 et $2,34 \mathrm{mg} / \mathrm{L}$ contre la valeur limite qui est de $0,02 \mathrm{mg} / \mathrm{L}$. La distribution de l'ammonium dans un milieu aquatique varie selon le niveau de productivité de l'écosystème et son degré de pollution par la présence de matière organique. Sa présence dans les eaux de la rivière résulterait de la dégradation aérobie de l'azote organique (protéines, acides aminés, urée...) lequel provient en grande partie des rejets des eaux non épurées. En effet, le $\mathrm{NH}^{+}$en lui-même n'est pas nuisible mais peut se transformer sous certaines conditions en ammoniac $\left(\mathrm{NH}_{3}\right)$, un gaz soluble dans l'eau et toxique pour la vie aquatique (BE, 2012). Hébert et Légaré (2000) démontrent que dans un milieu bien oxygéné, l'ammonium est rapidement utilisé et sa concentration est faible. Les teneurs retrouvées, ne présentent donc pas un risque pour la rivière Sô car celle-ci est bien oxygénée. Les concentrations en nitrate de la rivière sont comprises entre 1,20 et $3,33 \mathrm{mg} / \mathrm{L}$, ce qui excède la norme internationale fixée à $0,02 \mathrm{mg} / \mathrm{L} \quad(\mathrm{BE}, 2012)$. Ces concentrations élevées de nitrate s'expliquent prioritairement par la présence des déchets ménagers et commerciaux de toutes sortes produits par les riverains. Quant aux nitrites, elles présentent des concentrations comprises entre 0,09 et $0,20 \mathrm{mg} / \mathrm{L}$ contre une valeur normale de $0,02 \mathrm{mg} / \mathrm{L}$. Ces concentrations élevées peuvent affecter le développement des espèces aquatiques, car une eau renfermant des nitrites même à faible doses, peut être considérée comme suspecte, voire létale, pour les poissons (Vissin et al., 2010). Ces taux s'expliquent également par les conditions d'anaérobiose existants par endroits par l'abondance des macrophytes et favorisant une nitrification assez poussée des matières organiques. L'azote total présente des valeurs comprises entre 3,91 et $5 \mathrm{mg} / \mathrm{L}$ avec une moyenne de $4,56 \mathrm{mg} / \mathrm{L}$. Même s'il n'existe pas de critère de toxicité pour l'azote total, une concentration plus élevée que $1,0 \mathrm{mg} / \mathrm{L}$ dans les eaux de surface est considérée comme étant indicatrice d'une problématique de surfertilisation dans le milieu (Hébert et Légaré, 2000). Ceci indique donc un état de pollution critique et prédispose surtout les eaux du groupe II à des perturbations. S'agissant des composés phosphorés, les teneurs moyennes en orthophosphates

\section{CONCLUSION}

De nos jours, la sauvegarde et la protection des écosystèmes aquatiques sont d'une nécessité fondamentale pour la biodiversité des espèces halieutiques. L'étude spatio-temporelle de la qualité des eaux de la rivière Sô a permis de faire une caractérisation physico-chimique. Cette caractérisation montre que les et en phosphore total sont respectivement de $0,35 \mathrm{mg} / \mathrm{L}$ et de 1,33mg/L. En effet, les orthophosphates résultent de la dégradation des bactéries de phosphates organiques provenant du rejet d'eaux usées (métabolisme, poudres à lessiver, industries agro-alimentaires et chimiques) et de l'utilisation d'engrais. Assimilables par les plantes et organismes photosynthétiques, ils interviennent de façon déterminante dans les phénomènes d'eutrophisation des cours d'eau (BE, 2012). Toutefois, au-dessus d'une certaine concentration et lorsque les conditions sont favorables (faible courant, transparence adéquate.), il peut provoquer une croissance excessive d'algues et de plantes aquatiques (MEF, 2013). Les valeurs retrouvées dans les eaux de la rivière Sô sont supérieures aux valeurs tolérantes admises et traduisent donc l'apport excessif du lessivage et du ruissellement des terres agricoles fertilisées. Sur la rivière Sô, on note que les eaux sont beaucoup plus influencées par les différentes saisons (pluvieuse et sèche) de l'année. En effet, durant la grande saison pluvieuse et la petite saison sèche, les teneurs en sels nutritifs surtout azotés et phosphorés, deviennent importantes. Ces sels nutritifs sont drainés par les eaux de ruissellement chargées de produits de lessivage des bassins versants et par l'arrivée des eaux continentales, riches en matières organiques. Ce qui crée une pollution organique dans le cours inférieur et moyen de la rivière telle que démontré par la typologie abiotique réalisée. Elle est également liée à l'usage abusif des engrais chimiques lors des activités agricoles qui se développent le long du bassin versant de la rivière ainsi qu'à l'élevage des porcs et bœuf qui sont laissés en divagation laissant d'importantes quantités de déjections dans la rivière. Par contre ces différents facteurs de pollution sont moins voire absents au niveau du cours supérieur de la rivière qui est tout de même moins peuplé. Le rôle de ces éléments azotées et phosphorées dans le contrôle de la production a été souligné par plusieurs études (Trinqier, 2009 ; Mama et al., 2011; Noumon et al., 2015 ; Tchakonté et al., 2015 et Zinsou et al., 2016). Ils sont à surveiller fortement car représentent des grands facteurs d'eutrophisation des cours et plans d'eau.

principaux paramètres physiques (Température, $\mathrm{pH}$, Oxygène dissous, salinité, calcium et magnésium) présentent des valeurs relativement compatibles à la vie aquatique. Le suivi des concentrations en nutriments de la rivière révèle des teneurs élevées en composées 
azotées et phosphorées, responsables de l'eutrophisation des cours et plans d'eau.

La typologie abiotique montre que :

- le cours supérieur de la rivière présente des eaux bien oxygénées, transparentes mais accompagnée d'une simple pollution azotée ;

- le cours moyen présente une forte pollution azotée et phosphorée ;

- le cours inférieur présente des eaux fortement minéralisées associées également à une pollution azotée.

\section{REMERCIEMENTS}

Les auteurs remercient le Ministère de l'Enseignement Supérieur et de la Recherche Scientifique du Bénin pour son soutien financier et le technicien Lyde TOMETIN du

\section{REFERENCES BIBLIOGRAPHIQUES}

Adité A, Winemiller KO, Fiogbé ED, 2006. Population structrure and reproduction of the African bonytongue, Heterotis niloticus in the Sô River foodplain system (West Africa): implications for management. Ecology of Freshwater Fish 2006: 15: $30-39$.

Ahouanssou M, 2011. Diversité et exploitation des poissons de la rivière Pendjari (Bénin, Afrique de l'Ouest). Thèse de doctorat de l'Université d'Abomey-Calavi (Bénin), 216p.

Beaux JF, 1998. L'environnement, Repères pratiques, Nathan, 160p.

Belaud A, 1987. Inland water quantity and quality. ENSA. Toulouse/Applied Ichthyology.

Belkhiri L, Boudoukha A, Mouni L, Baouz T, 2010. Multivariate statistical characterisation of groundwater quality in Ain Azel plain, Algeria. African Journal of Environmental Science and Technology.Vol.4(8), pp.526-534.

Bonou C et Adisso P, 2002. Evaluation of organic and bacteriological pollution excreta, wastewater and solid waste in the lagoon of Cotonou, CPU/UAC.

Bruxelles Environnement (BE), 2012. Programmes de surveillance de l'état des eaux de la Région de Bruxelles-Capitale en application de l'Ordonnance Cadre Eau.

Chouti W, Mama D et Alapini F, 2010. Études des variations spatio-temporelles de la pollution des eaux de la lagune de Porto-Novo (Sud-Bénin). Journal of Applied Biosciences 4 (4): 1017-1029.

Conseil Canadien des Ministres de l'Environnement (CCME), 2011. Manuel des protocoles d'échantillonnage pour l'analyse de la qualité de l'eau au Canada. In CCME, Publications-Eau.
Ces résultats montrent l'urgence de la mise en place d'un système qui contrôle et traite les eaux usées avant leur déversement dans la rivière Sô ainsi qu'un contrôle de l'usage des engrais sur tout le bassin versant de la rivière et de l'encadrement des porcs et bovins laissés en divagation sur ses berges. Afin de préserver les usages que procure cette rivière aux populations riveraines, il importe qu'une approche intégrée de la gestion de celle-ci et de son bassin versant soit instaurée pour sa protection et les organismes vivants qui y habitent.

Laboratoire de Chimie Inorganique pour sa contribution aux analyses de Laboratoire.

Dèdjiho C. A., Mama D., Tomètin L., Nougbodé I., Chouti W.,Sohounhlouè D.C.K. et Boukari M., 2013. Évaluation de la qualité physico-chimique de certains tributaires d'eaux uses du lac Ahémé au Bénin. Journal of Applied Biosciences 70 : 56085616.

Hébert $S$ et Légaré S, 2000. Suivi de la qualité des rivières et petits cours d'eau, Québec, Direction du suivi de l'état de l'environnement, ministère de l'environnement, envirodoq $n^{\circ}$ ENV-20010141, rapport $n^{\circ} \mathrm{QE}-124,24 p$ et 3 annexes.

Kiossa C, 2011. Biodiversité et exploitation de la rivière de la rivière Sô au Bénin. Mémoire de fin d'étude de Master, Université d'Abomey-Calavi, $102 \mathrm{p}$.

Lalèyè $P, 1995$. Écologie comparée de deux espèces de Chrysichthys, poissons Siluriformes (Claroteidae) du complexe lagunaire « Lac Nokoué-Lagune de Porto-Novo » au Bénin. Thèse de doctorat, Université de Liège, 199p.

Mama D, Chouti W, Alassane A, Changotade O, Alapini $F$ et Boukari M, 2011. Étude dynamique des apports en éléments majeurs et nutritifs des eaux de la lagune de Porto-Novo (Sud-Bénin). Int J. Chem. Sci 5 (3) :1278-1293.

Ministère de l'Environnement et de la Faune du Québec (MEF), 2013. Pour une eau de qualité en milieu rural, comprendre et agir collectivement, ministère de du Québec, Direction des écosystèmes aquatiques, 387p.

Nisbet M. et Verneaux J, 1970. Composantes chimiques des eaux courantes. Annales de Limnologie, tome 6. 161-190.

Noumon CJ, Mama D, Dedjiho CA, Agbossou E et Ibouraima S, 2015. Evaluation de la qualité 
physico-chimique et du risque d'eutrophisation de la retenue d'eau de Kogbétohouè (SudBénin). Journal of Applied Biosciences 85: 78487861.

Onana F, Zébazé T, Nyamsi T, Domche T et Ngassam P, 2014. Distribution spatio-temporelle du zooplancton en relation avec les facteurs abiotiques dans un hydrosystème urbain : le ruisseau de Kondi, Cameroun. Journal of Applied Biosciences 82: 7326-7338, 13p.

Pouomogne V, 1998. Pisciculture en Milieu Tropical Africain. Comment produire du poisson à côut modéré. Presse Universitaire d'Afrique, Yaoundé, 263p.

Rodier J, 2009, L'analyse de l'eau. Dunod, gème édition, $1526 \mathrm{p}$.

Tachet $\mathrm{H}$, Bournaud M, Richoux P et Usseglio-Polatera $P, \quad 2000$. Invertébrés d'eau douce: systématique, biologie, écologie. CNRS édition, Paris : 587.

Tchakonté S, Ajeagah G, Diomandé D, Camara A, Konan $M$ et Ngassam P, 2015. Impact of anthropogenic activities on water quality and Freshwater Shrimps diversity and distribution in five rivers in Douala, Cameroun. Journal of Biodiversity and Environmental Sciences 4: 183-194.

Trinquier C, 2009. Le risque d'eutrophisation des lagunes méditerranéennes: le cas de la lagune de Thau(Hérault). Master 1 professionnel Gestion des catastrophes et des risques naturels. Université Paul Valéry-montpellier III UFR III, $121 \mathrm{p}$.

Vissin E, Sintondji L et Houssou C, 2010. Étude de la pollution des eaux et de la contamination du Tilapia guineensis du chenal de Cotonou par le plomb. RGLL, $n^{\circ} 08$.

Zinsou HL, Attingli AH, Gnonhossou P, Adandédjan D et Lalèyè $P, 2016$. Caractéristiques physicochimiques et pollution de l'eau du Delta de I'Ouémé au Bénin. Journal of Applied Biosciences 97: 9163-9173. 